\title{
Medication exposure and predictors of first mood episode in offspring of parents with bipolar disorder: a prospective study
}

\author{
Fabiano G. Nery, ${ }^{1}$ iD Anna R. Wilson, ${ }^{1}$ Marguerite R. Schneider, ${ }^{2}$ Jeffrey R. Strawn, ${ }^{1}$ Luis R.
} Patino, ${ }^{1}$ Robert K. McNamara, ${ }^{1}$ Caleb M. Adler, ${ }^{1}$ Stephen M. Strakowski, ${ }^{3}$ Melissa P. DelBello ${ }^{1}$

${ }^{1}$ Department of Psychiatry and Behavioral Neuroscience, University of Cincinnati College of Medicine, Cincinnati, OH, USA. ${ }^{2} \mathrm{Harvard}$ Longwood Psychiatry Residency Training Program, Boston, MA, USA. ${ }^{3}$ Department of Psychiatry, Dell Medical School, University of Texas at Austin, Austin, TX, USA.

\begin{abstract}
Objectives: To prospectively investigate whether baseline clinical characteristics and medication exposure predict development of major depressive disorder or bipolar disorder in offspring of parents with bipolar disorder.

Methods: Youth aged 9-20 years with at least one biological parent with bipolar disorder and no prior history of mood or psychotic episodes $(n=93)$ were prospectively evaluated and treated naturalistically during the study. Participants were divided into two groups: converters, defined as those who met DSM-IV criteria for a mood episode during follow-up $(n=19)$; or non-converters $(n=74)$. Logistic regression models were used to investigate associations between baseline clinical variables and medication exposure during follow-up and risk of developing a first mood episode (conversion).

Results: Multivariate regression analyses showed that baseline anxiety disorders and subsyndromal mood disorders were associated with increased risk of conversion during follow-up. Adding medication exposure to the multivariate model showed that exposure to antidepressants during follow-up was associated with increased risk of conversion.

Conclusions: Caution should be used when treating bipolar offspring with anxiety and/or emerging depressive symptoms using antidepressant agents, given the increased risk of developing a major mood disorder.
\end{abstract}

Keywords: Bipolar disorder; mood disorders; risk factors; antidepressive agents

\section{Introduction}

Children and adolescent offspring of parents with bipolar disorder (bipolar offspring) are at increased risk of developing psychiatric disorders ${ }^{1,2}$ - including anxiety, substance use, disruptive behavior, and sleep disorders. ${ }^{1,3,4}$ The risk is especially high for the development of mood disorders, which affect up to $67 \%$ of these youth. ${ }^{1,3,4}$ Regarding bipolar disorders specifically, one study found that $13 \%$ of bipolar offspring developed bipolar disorder spectrum disorders, while 3\% met criteria for DSM-IV bipolar disorder type $\mathrm{I}^{5}$ a rate that is higher than that observed in the general population.

Among bipolar offspring, symptoms of bipolar disorder often first manifest during adolescence, ${ }^{1,3,4}$ making this age period a crucial time to implement preventative and treatment intervention strategies. Bipolar offspring who remain asymptomatic through childhood and early to midadolescence are more likely to remain well during later

Correspondence: Melissa P. DelBello, University of Cincinnati, Department of Psychiatry and Behavioral Neuroscience, 260 Stetson St. Suite, 3200 Cincinnati, OH 45219, USA.

E-mail: delbelmp@ucmail.uc.edu

Submitted Nov 26 2019, accepted Mar 01 2020, Epub May 112020. periods, ${ }^{6}$ suggesting a dramatic decrease in the risk of psychiatric disorders after a symptom-free adolescence in this group.

Given the potential for preventative or therapeutic intervention during this sensitive developmental period, many recent studies have investigated risk factors for mood disorders among youth bipolar offspring. Increasing evidence suggests that anxiety (as either a dimensional symptom or a categorical diagnosis), emotional lability, subthreshold manic or hypomanic symptoms, depressive symptoms, or minor depressive diagnoses are all associated with later conversion to a mood disorder, in general, or to a bipolar disorder specifically. ${ }^{4,7-9}$ In at least some of these contexts, the symptoms might represent prodromal manifestations of bipolar disorder rather than a comorbidity. ${ }^{10}$ Other efforts have included the development of an individual risk calculator to predict the 5-year risk of developing bipolar disorder in bipolar offspring. ${ }^{11}$ This risk calculator showed that anxiety, manic symptoms, depressive
How to cite this article: Nery FG, Wilson AR, Schneider MR, Strawn JR, Patino LR, McNamara RK, et al. Medication exposure and predictors of first mood episode in offspring of parents with bipolar disorder: a prospective study. Braz J Psychiatry. 2020; 42:481-488. http://dx.doi.org/10.1590/1516-4446-2019-0802 
symptoms, mood lability, poor general psychosocial functioning, and earlier parental age at onset collectively predict the emergence of bipolar disorder among bipolar offspring.

Despite the fact that anxiety, subsyndromal manic or depressive symptoms, and mood lability often require psychopharmacological treatment, the effects of psychotropic medication on accelerating or delaying the onset of mood disorders among bipolar offspring are poorly understood. It has been hypothesized that, in bipolar offspring, antidepressants and psychostimulants may decrease the age at which bipolar disorder would first manifest. ${ }^{12}$ For instance, exposure to antidepressants and stimulants has been associated with greater prevalence of bipolar disorder among offspring of parents with bipolar disorder. ${ }^{13}$ In addition, our group has reported that $57 \%$ of youth bipolar offspring exposed to antidepressants exhibited adverse reactions, including irritability, aggression, impulsivity, or hyperactivity, which led to treatment discontinuation; the risk of adverse reactions was greater when medication treatment was initiated at a younger age. ${ }^{14}$ In contrast, other studies have not found an association between exposure to antidepressants and stimulants and age of onset of bipolar disorder among bipolar offspring. ${ }^{15}$

With these considerations in mind, we prospectively investigated associations between baseline demographic and clinical characteristics and medication exposure as risk factors for developing a first mood episode among bipolar offspring. Based on prior research, we hypothesized that subthreshold mood disorders and anxiety disorders, as well as exposure to antidepressants and stimulants, would be associated with the development of major depressive disorder or bipolar disorder among bipolar offspring.

\section{Methods}

\section{Study participants}

This report presents clinical findings from a larger longitudinal multimodal imaging study conducted from 2007 to $2015 .^{16-18}$ Subjects were included if they were 9-20 years old and had at least one biological parent with bipolar disorder (type I) confirmed by the Structured Clinical Interview for the DSM-IV (SCID). ${ }^{19}$ Subjects were excluded if they had baseline DSM-IV diagnoses of a mood or psychotic disorder including bipolar disorder I or II disorders, cyclothymia, dysthymia, schizophrenia, schizoaffective disorder, psychosis not otherwise specified (NOS), or a history of alcohol or drug dependence. Illnesses not fully developed, including depressive disorder or bipolar disorder NOS, were permitted. We assessed Axis I disorders using the Washington University Kiddie-Schedule for Affective Disorders and Schizophrenia Lifetime Version (K-SADS-P/L) and the mood disorders section of the WASH-U KSADS. ${ }^{20,21}$ Youth were also excluded if they had a history of mental retardation, defined as having an estimated intelligence quotient (IQ) score < 70 confirmed with the Wechsler Abbreviated Scale of Intelligence (WASI).

\section{Ratings and assessments}

Baseline assessments included demographic variables, psychiatric and medical history, medication exposure history, IQ assessment, and assessment of mood symptoms. Depressive and manic symptoms were respectively assessed with the 17-item Hamilton Depression Rating Scale $(\mathrm{HAMD})^{22}$ and the Young Mania-Rating Scale (YMRS). ${ }^{23}$ Clinical global severity was assessed with the Clinical Global Impression Scales for Severity and Improvement (CGI-S and CGI-I respectively). ${ }^{24}$ To ensure diagnostic and rating reliability, all scales were administered by trained raters with established reliability (all diagnostic $\kappa$ and intraclass correlation coefficients [ICC] $>0.9$ ).

Depending on symptom severity, repeated evaluations were performed every 1-4 months after the baseline visit, for a mean follow-up time of 140.6 weeks (range = 1-407, standard deviation [SD] = 88.7) for included subjects. Treatment throughout the study was naturalistic and information regarding exposure to psychotropic medications was obtained at each visit. The presence of mood symptoms was also assessed at each follow-up visit using a modified version of the Longitudinal Interval Follow-up Evaluation (LIFE) Interview, which allowed for week-toweek estimates of mood symptom severity throughout the study. ${ }^{25}$

For the present analysis, we excluded all subjects who missed any follow-up visit after the baseline assessment. All subjects with at least one follow-up visit were included. The subjects were divided into two groups for analysis: converters, who met full DSM-IV-TR criteria for a major depressive, manic, mixed or hypomanic episode at any time during follow-up, and non-converters who did not meet criteria for a mood episode during follow-up.

\section{Statistical analysis}

Initial analysis focused on baseline demographic and clinical characteristics including age, sex, race, IQ, socioeconomic status (SES), psychiatric diagnoses, depressive and manic symptom rating scale scores, and prior exposure to stimulants (the use of medications from classes other than stimulants was not sufficiently frequent to allow valid statistical analyses of their effects on risk of developing a first mood episode). DSM-IV-TR psychiatric diagnoses present at baseline were categorized as follows: attention-deficit/hyperactivity disorder (ADHD), mood disorder NOS (which included depressive disorder NOS and bipolar disorder NOS), anxiety disorders (including generalized anxiety disorder, post-traumatic stress disorder, separation anxiety disorder, simple phobia, and social phobia), and disruptive behavioral disorders (including oppositional defiant disorder and conduct disorder). Baseline age, IQ, SES, YMRS, and HAMD were considered continuous variables. Sex, race, baseline psychiatric diagnoses, and prior stimulant exposure were considered categorical binary variables. Race (self-reported) was coded as white vs. non-white because the sample was predominantly white $(n=66,71 \%)$ and no other racial group was large enough (African Americans, $\mathrm{n}=20[21.5 \%]$, Hispanic, $\mathrm{n}=1[1.1 \%]$, others (subjects 
identifying as multi-racial), $n=6[6.5 \%])$ to support individual consideration.

Medications of interest were categorized into four distinct types: antidepressants, stimulants, mood stabilizers (lithium and/or an anticonvulsants), and secondgeneration antipsychotics. The effects of medications were evaluated using both binary and continuous measures including exposure during follow-up (yes or no), duration of exposure during follow-up (in weeks), and percentage of follow-up weeks on medication. Psychotherapy during follow-up was treated as a categorical variable (yes or no) or as a continuous variable (total number of psychotherapy sessions during follow-up).

Simple logistic regression using standard maximum likelihood methods was used to explore the relationship between individual demographic, baseline clinical, and follow-up medication exposure variables and the risk of developing a first mood episode among bipolar offspring.

Multiple logistic regression with stepwise forward selection was used to explore the potential multivariate models. Two potential multivariate models were considered, one including only demographic and clinical variables present at baseline, and one including both baseline variables and medication exposure during the follow-up period. Using a cutoff for initial inclusion in the model of $p<0.25$ in the univariate regression models, baseline variables considered for the multiple regression model were age, sex, SES, depressive disorder NOS, anxiety disorder (any), and ADHD. Using the same cutoff, follow-up variables related to medication exposure were antidepressant exposure during follow-up, mood stabilizer exposure during follow-up, atypical antipsychotic exposure during follow-up, and psychotherapy exposure during follow-up. Despite the statistical association between baseline HAMD and YMRS scores and variables related to medication use during follow-up (such as duration of use and percentage of weeks using medication) or number of psychotherapy sessions and risk of conversion, these variables were not included in the model due to their negligible effect (as demonstrated by their exp[B] or odds ratio [OR] in univariate analysis) and their collinearity (which was explored by bivariate correlation analyses, with $r$ values ranging from 0.33 to 0.55 , $p$-values ranging from $p=0.001$ to $p<0.001$ for baseline variables, and $r$ values ranging from 0.7 to 0.9 , with $p<0.001$ for medication exposure variables).

Statistical analyses were conducted using the IBM SPSS version 2; $p$ values $<0.05$ were considered statistically significant.

\section{Ethics statement}

All subjects and their legal guardians provided written informed consent/assent after study procedures were fully explained. The study was approved by the University of Cincinnati institutional review board.

\section{Results}

Of the original 122 subjects who had a baseline assessment, 29 missed all follow-up visits and were excluded from this analysis. Therefore, the final study population included 93 individuals, 44 (47.3\%) boys and 49 (52.7\%) girls, with a mean age of $13.7 \pm 2.9$ years at baseline. The subjects were divided into two groups, converters $(n=19)$, who met full DSM-IV-TR criteria for a major depressive, manic, mixed or hypomanic episode at any time during follow-up, and non-converters $(n=74)$, who did not meet criteria for a mood episode during follow-up. No subjects developed a psychotic disorder. Baseline demographic and clinical characteristics of the converters and nonconverters are displayed in Table 1. Subjects lost to follow-up after baseline visit were not statistically different from converters and non-converters regarding baseline demographic and clinical characteristics, including age, sex, race, pubertal status, IQ, SES, clinical diagnoses, and YMRS mean scores. Subjects lost to follow-up had significantly lower baseline HAMD scores (mean \pm SD = $5.6 \pm 5.2)$ than converters $(p<0.001)$, but similar to those of non-converters $(p=0.38)$. Participants who were lost to follow-up were also less likely to be medicated at baseline, or have baseline childhood-onset psychiatric diagnoses.

Of the 19 bipolar offspring converters, 12 had a major depressive episode and seven had a manic or mixed episode. From baseline, the mean time to develop a mood episode was 193 weeks (median: 197 weeks; range 71-344 weeks). The mean (SD) age for developing a first mood episode was 16.5 (2.4) years (range: 13.5 to 23 years old). The mean follow-up of 74 bipolar offspring who did not develop a first mood episode was 126 (88) weeks (median: 105 weeks, range: 1-407 weeks).

Converters were more likely to be girls $(p=0.04)$ and of lower SES $(p=0.09)$. They also had higher baseline depressive symptom scores $(p=0.003)$ and were more likely to have anxiety disorders $(p=0.02)$ and mood disorder NOS $(p<0.001)$ at baseline. During follow-up, converters also had overall higher rates of exposure to antidepressants $(p<0.001)$ and mood stabilizers $(p=0.05)$ than non-converters. They also had higher duration of use and percentage of time using antidepressants, secondgeneration antipsychotics, and mood stabilizers ( $p$-values ranging from 0.05 to $<0.001$ ). Converters were also more likely to receive psychotherapy $(p=0.03)$ and to have higher number of psychotherapy sessions during follow-up $(p=0.002)$ (Table 2).

\section{Univariate logistic regression}

Table 3 show the results of univariate logistic regression analysis for baseline variables. Anxiety disorder (any), mood disorder NOS, and baseline HAMD scores significantly predicted conversion during follow-up. Bipolar offspring with an anxiety disorder were more likely to convert than those without an anxiety disorder: $32 \%$ of those with an anxiety disorder at baseline converted, while only $11 \%$ of those without an anxiety disorder converted $(p=0.02)$. Baseline mood disorder NOS was highly predictive of conversion during follow-up. Bipolar offspring with mood disorder NOS at baseline had seven times higher risk of converting during follow-up than those without baseline mood disorder NOS. The higher the 
Table 1 Baseline demographic and clinical characteristics of the sample

\begin{tabular}{|c|c|c|c|}
\hline Baseline characteristics & Converters $(n=19)$ & Non-converters $(n=74)$ & $p$-value \\
\hline Age (years) & $13.1(2.0)$ & $13.9(3.0)$ & 0.25 \\
\hline Girls, n (\%) & $14(74)$ & $35(47)$ & 0.04 \\
\hline White race, $\mathrm{n}(\%)$ & $14(74)$ & $52(70)$ & 0.71 \\
\hline Tanner stage, growth & $3.2(0.8)$ & $3.3(1.2)$ & 0.93 \\
\hline Tanner stage, pubic & $3.3(0.9)$ & $3.3(1.2)$ & 0.97 \\
\hline IQ & $101.9(11.3)$ & $104.3(18.5)$ & 0.59 \\
\hline SES & $35.3(10.1)$ & $39.5(8.8)$ & 0.08 \\
\hline HAMD & $11.7(5.7)$ & $6.8(6.4)$ & 0.003 \\
\hline YMRS & $9.1(4.9)$ & $6.9(6.0)$ & 0.15 \\
\hline Follow-up (weeks) & $193.4(3.0)$ & 127.0 & 0.003 \\
\hline \multicolumn{4}{|l|}{ Psychiatric diagnoses, n (\%) } \\
\hline Anxiety disorders (any) & $6(32)$ & $8(11)$ & 0.02 \\
\hline ADHD & $4(21)$ & $28(38)$ & 0.28 \\
\hline Mood disorders NOS & $9(48)$ & $8(11)$ & $<0.001$ \\
\hline Disruptive behavioral disorders & $2(11)$ & $4(5)$ & 0.6 \\
\hline Lifetime exposure to antidepressants, $n(\%)$ & $0(0)$ & $4(5)$ & - \\
\hline Lifetime exposure to stimulants, $\mathrm{n}(\%)$ & $3(16)$ & $14(19)$ & 0.75 \\
\hline
\end{tabular}

Data presented as mean (standard deviation), unless otherwise specified.

$\mathrm{ADHD}=$ attention-deficit/hyperactivity disorder; HAMD = Hamilton Depression Rating Scale; $\mathrm{IQ}=$ intelligence quotient; NOS = not otherwise specified; SES = socioeconomic status; YMRS = Young Mania Rating Scale.

Table 2 Characteristics of exposure to treatment during follow-up

\begin{tabular}{|c|c|c|c|}
\hline Treatment & Converters $(n=19)$ & Non-converters $(n=74)$ & $\mathrm{p}$-value \\
\hline \multicolumn{4}{|l|}{ Antidepressants } \\
\hline Exposure, $\mathrm{n}(\%)$ & $13(68)$ & $16(22)$ & $<0.001$ \\
\hline Duration of use (weeks) & $36.4(44.0)$ & $13.4(38.3)$ & 0.03 \\
\hline Percentage of time in use & $21.0(27.0)$ & $8.7(21.9)$ & 0.04 \\
\hline \multicolumn{4}{|l|}{ Stimulants } \\
\hline Exposure, n (\%) & $10(53)$ & $28(38)$ & 0.24 \\
\hline Duration of use (weeks) & $42.1(56.1)$ & $30.0(56.6)$ & 0.4 \\
\hline Percentage of time in use & $22.5(32.4)$ & $20.0(34.8)$ & 0.78 \\
\hline \multicolumn{4}{|c|}{ Second-generation antipsychotics } \\
\hline Exposure, $\mathrm{n}(\%)$ & $7(37)$ & $13(18)$ & 0.11 \\
\hline Duration of use (weeks) & $33.3(51.5)$ & $4.0(11.3)$ & $<0.001$ \\
\hline Percentage of time in use & $16.8(24.1)$ & $5.3(17.1)$ & 0.02 \\
\hline \multicolumn{4}{|l|}{ Mood stabilizers } \\
\hline Exposure, $\mathrm{n}(\%)$ & $4(21)$ & $4(5)$ & 0.05 \\
\hline Duration of use (weeks) & $10.6(24.0)$ & $1.1(5.3)$ & 0.002 \\
\hline Percentage of time on use & $6.0(13.3)$ & $1.2(6.2)$ & 0.02 \\
\hline \multicolumn{4}{|l|}{ Psychotherapy } \\
\hline Exposure, n (\%) & $17(89.5)$ & 47 (63.5) & 0.03 \\
\hline Number of sessions & $34.8(42.4)$ & $11.1(23.8)$ & 0.002 \\
\hline
\end{tabular}

Data presented as mean (standard deviation), unless otherwise specified.

baseline HAMD score, the higher the risk of conversion during follow up.

Table 4 describes the findings from the univariate logistic regression model of medication exposure. With the exception of stimulant exposure, all variables associated with medication and psychotherapy exposure were significantly associated with risk of developing a mood episode during follow-up.

\section{Multivariate logistic regression}

The first multivariate logistic regression model, which included only baseline variables (age, sex, SES, anxiety disorder [any], mood disorders, and ADHD), showed that only anxiety disorders and mood disorders emerged as significant (Table 5). This model was statistically significant $\left(\chi^{2}=14.7, p=0.001\right.$, Nagelkerke $\left.R^{2}=0.24\right)$, correctly classifying $80.9 \%$ of the cases. In this model, participants with baseline anxiety disorders were 4.4 times more likely to convert during follow-up, and those with baseline mood disorder NOS were 7.8 times more likely to convert during follow-up.

A second multivariate model, which added medication and psychotherapy exposure variables to the abovementioned baseline variables, was statistically significant, with a $\chi^{2}=27.9, p<0.001$, Nagelkerke $R^{2}=0.43$, and 
correctly classified $85.4 \% \%$ of the cases. In this model, female sex and lower SES were both associated with a slight increase in risk of conversion during follow-up. Participants with a baseline mood disorder NOS were 7.8 times more likely to convert, and participants exposed to antidepressant treatment during follow-up were 6.6 times more likely to convert during follow-up (Table 5). Baseline anxiety disorders and exposure to mood stabilizers and atypical antipsychotics were not associated with increased risk for conversion in this model.

Given that baseline mood disorder NOS and antidepressant exposure during follow-up could be interacting with each other (i.e., participants with baseline mood

Table 3 Simple logistic regression analysis of association between baseline demographic and clinical variables and conversion to a mood episode during follow-up in bipolar offspring

\begin{tabular}{lccc}
\hline Variables & OR & p-value & $95 \% \mathrm{Cl}$ \\
\hline Baseline demographic variables & & & \\
Age & 0.9 & 0.25 & $0.7-1.1$ \\
Sex & 0.32 & 0.05 & $0.11-0.98$ \\
Race & 1.0 & 0.98 & $0.49-2.3$ \\
IQ & 0.99 & 0.58 & $0.98-1.0$ \\
SES & 0.95 & 0.09 & $0.9-1.0$ \\
Handedness & 2.8 & 0.28 & $0.4-18.0$ \\
& & & \\
Baseline clinical variables & & & \\
HAMD & 1.12 & 0.005 & $1.04-1.2$ \\
YMRS & 1.1 & 0.15 & $0.98-1.2$ \\
Anxiety disorder (any) & 3.8 & 0.03 & $1.1-12.8$ \\
Mood disorder NOS & 7.4 & 0.001 & $2.3-23.7$ \\
Disruptive disorders & 2.0 & 0.43 & $0.39-12.2$ \\
ADHD & 0.44 & 0.18 & $0.13-1.5$ \\
Prior exposure to stimulants & 0.8 & 0.75 & $0.2-3.1$ \\
\hline
\end{tabular}

$95 \% \mathrm{Cl}=95 \%$ confidence interval; ADHD = attention-deficit/ hyperactivity disorder; HAMD = Hamilton Depression Rating Scale; $\mathrm{IQ}=$ intelligence quotient; NOS = not otherwise specified; OR = odds ratio; SES = socioeconomic status; YMRS = Young Mania Rating Scale. disorder being more likely to receive antidepressant treatment during follow-up), we explored possible interactions between these two variables by consecutively removing each of them from the multivariate model. When removing baseline mood disorder NOS, antidepressant exposure during follow-up remained as a significant predictor of conversion, with slight increase in risk estimate $(\mathrm{OR}=7.5$, 95\% confidence interval $(95 \% \mathrm{Cl})$ 2.4-23.2, $p=0.001$ ), whereas female sex and SES lost significance as predictors of conversion. As expected, removing antidepressant exposure during follow-up yielded similar results to the model including only baseline variables.

\section{Discussion}

Despite the current knowledge regarding demographic and clinical variables that are associated with risk of developing major depressive disorder or bipolar disorder among bipolar offspring, , $4-9,13,26-28$ and the recent attempts to predict this risk at an individual level, ${ }^{11}$ little is known about the contribution of medication exposure to the risk of developing bipolar disorder in those at familial risk.

Consistent with our hypothesis, our results suggest that antidepressant exposure may increase the risk of conversion in bipolar offspring regardless of the presence of subthreshold mood symptoms at baseline. This finding is consistent with a prior hypothesis that antidepressants may carry deleterious effects in bipolar offspring, ${ }^{12}$ put forth due to increased rates of bipolar disorder diagnosis among bipolar offspring treated with antidepressants, ${ }^{13,15}$ and increased rates of adverse events from antidepressant treatment in bipolar offspring treated for depressive disorder NOS or anxiety disorders. ${ }^{14}$ In contrast with these previous studies, ${ }^{13,15}$ which were cross-sectional and thus not capable of detecting the temporal association between medication exposure and mood disorder development, our longitudinal study advances the field by tracking the beginning of antidepressant treatment

Table 4 Simple logistic regression analysis of association between exposure to treatment variables and conversion to a mood episode during follow-up in bipolar offspring

\begin{tabular}{|c|c|c|c|}
\hline Treatment variables & OR & $p$-value & $95 \% \mathrm{Cl}$ \\
\hline Antidepressant use during follow-up & 7.9 & $<0.001$ & $2.6-23.9$ \\
\hline Duration of antidepressant use during follow-up (weeks) & 1.01 & 0.04 & 1.0-1.02 \\
\hline Percentage of follow-up weeks on antidepressants & 1.02 & 0.05 & $1.0-1.04$ \\
\hline Lifetime exposure to antidepressants & 6.2 & 0.001 & 2.1-18.4 \\
\hline Stimulant use during follow-up & 2.1 & 0.17 & $0.7-5.7$ \\
\hline Duration of stimulant use during follow-up (weeks) & 1.0 & 0.4 & $0.99-1.01$ \\
\hline Percentage of follow-up weeks on stimulants & 1.0 & 0.78 & $0.98-1.02$ \\
\hline Lifetime exposure to stimulants & 1.5 & 0.47 & $0.5-4.0$ \\
\hline Mood stabilizer use during follow-up & 4.7 & 0.04 & $1.1-20.8$ \\
\hline Duration of mood stabilizer use during follow-up (weeks) & 1.01 & 0.03 & $1.0-1.1$ \\
\hline Percentage of follow-up weeks mood stabilizers & 1.01 & 0.05 & $1.0-1.1$ \\
\hline Lifetime exposure to mood stabilizers & 4.7 & 0.04 & $1.3-20.8$ \\
\hline Second-generation antipsychotic use during follow-up & 2.7 & 0.08 & $0.9-8.3$ \\
\hline Duration second-generation antipsychotic use during follow-up (weeks) & 1.0 & 0.002 & $1.0-1.1$ \\
\hline Percentage of follow-up weeks on atypical antipsychotics & 1.1 & 0.03 & $1.0-1.1$ \\
\hline Lifetime exposure to atypical antipsychotics & 3.0 & 0.05 & $0.98-9.2$ \\
\hline Psychotherapy during follow-up & 4.9 & 0.04 & $1.1-22.8$ \\
\hline Total number of psychotherapy sessions during follow-up & 1.0 & 0.008 & $1.01-1.04$ \\
\hline
\end{tabular}

$95 \% \mathrm{Cl}=95 \%$ confidence interval; OR (odds ratio). 
Table 5 Multiple logistic regression analysis of association between baseline and follow-up variables and conversion to a mood episode in bipolar offspring

\begin{tabular}{lccc}
\hline Variables & OR & p-value & $95 \% \mathrm{Cl}$ \\
\hline Model with only baseline variables & & & \\
$\quad$ Depressive disorder NOS & 7.8 & 0.002 & $2.2-27.9$ \\
Anxiety disorder (any) & 4.4 & 0.03 & $1.1-16.7$ \\
& & & \\
Model with baseline and follow-up & & & \\
variables & & & \\
Sex & 0.2 & 0.03 & $0.05-0.9$ \\
SES & 0.93 & 0.04 & $0.9-1.0$ \\
Depressive disorder NOS & 7.8 & 0.007 & $1.7-34.8$ \\
Antidepressant exposure during & 6.6 & 0.005 & $1.8-24.2$ \\
follow-up & & & \\
\hline
\end{tabular}

$95 \% \mathrm{Cl}=95 \%$ confidence interval; NOS = not otherwise specified $\mathrm{OR}=$ odds ratio; SES = socioeconomic status.

after baseline assessment and before the onset of a full mood episode.

In the present study, treatment was naturalistic. Therefore, the need for psychopharmacological treatment or psychotherapy might have been higher in participants at higher risk of conversion; therefore, the associations seen in the univariate analyses between mood stabilizers, second-generation antipsychotics, antidepressants, psychotherapy, and risk of conversion might be simply reflecting this fact. To limit the confounding effects of common psychopathologies on the risk of conversion, we adjusted our analyses for baseline anxiety disorders and mood disorders NOS. Our final multivariate model suggests that, after controlling for baseline psychopathology, only antidepressants were associated with increased risk of conversion. These findings highlight the need for further research on alternative interventions for treating anxiety and depressive disorders in high-risk youth, especially those with anxiety and depressive disorders, for which an antidepressant is a first-line treatment. Recent evidence suggests that psychotherapeutic interventions such as family-focused therapy or mindfulness-based therapy might be a good option to treat bipolar offspring without deleterious effects on affective illness course. ${ }^{29,30}$

Biological mechanisms underlying possible deleterious effects of antidepressants in bipolar offspring are unknown. Previous studies suggest that a dysfunction in a prefrontallimbic circuit through impaired top-down prefrontal regulation of bottom-up limbic hyperactivity is associated with aberrant emotional arousal. ${ }^{31,32}$ Studies in adults and youth suggest that antidepressants exert their therapeutic effect by normalizing this aberrant pattern of functional activation. ${ }^{33,34}$ However, antidepressants such as selective serotonin reuptake inhibitors were shown to initially increase amygdala activity, ${ }^{35,36}$ which might cause an early increase in anxiety and agitation during the initial stages of treatment. Therefore, it is possible that antidepressants may potentiate rather than attenuate a limbic system that is primed for reactivity in bipolar offspring who, in turn, are more prone to antidepressant-related adverse events. ${ }^{37,38}$ Other explanations include modified gene expression of monoamine transporters influencing affective stimuli processing in corticolimbic circuits, causing some youth to be more prone to adverse events, ${ }^{39}$ limbic hyperactivity in an acute and dose-dependent fashion, with adverse event-related limbic hyperactivity occurring with acute dose escalations and at higher doses, ${ }^{35,36}$ and sensitive periods in development when youth might be more vulnerable to antidepressant-related adverse events because of pruning of neural networks during adolescence. ${ }^{40,41}$

Another relevant finding of this study was the lack of association between conversion and ADHD, prior stimulant exposure, or stimulant exposure during follow-up. The literature is inconsistent regarding the effects of stimulant exposure in high-risk youth, with some studies reporting an association between bipolar disorder diagnosis and prior exposure to stimulants ${ }^{13,42}$ which is not observed in others. ${ }^{15}$ Our population, which excluded those who had already experienced a mood episode, may not be representative of the entire bipolar offspring group, as ADHD symptoms begin in early childhood, and bipolar offspring experiencing a manic reaction to stimulants following earlier exposure would not have been eligible. Our finding of lacking association between ADHD and conversion is consistent with the literature showing that ADHD may not be a risk factor for development of mood disorders in bipolar offspring. ${ }^{43}$ This suggests that bipolar offspring have higher rates of ADHD, but that ADHD does not increase risk of conversion.

Our finding showing that those with anxiety disorders at baseline were more likely to convert is consistent with previous studies ${ }^{4,7,13,27}$ suggesting that anxiety is associated with poorer outcomes in high-risk youth, and supports the notion of bipolar offspring with anxiety as an ultra-high-risk group that warrants close monitoring. However, this association may not be specific to bipolar offspring, as most prospective studies of youth suggest that having an anxiety disorder early in childhood and adolescence increases the risk of developing mood disorders. Additionally, it is noteworthy that adolescents with first episode of mania and comorbid anxiety disorders are less likely to remit and more likely to relapse. ${ }^{44}$ Further research is necessary to assess treatment strategies that aid in preventing progression to a mood disorder in this ultra-high-risk group.

Both higher baseline HAMD scores and subsyndromal mood disorder diagnoses increased risk of conversion, although the risk estimate for baseline HAMD scores was negligible. In contrast, the presence of baseline subthreshold mood disorder (depressive disorder NOS and bipolar disorder NOS) was associated with higher risk of conversion both in univariate and multivariate analyses. Consistent with our findings, subthreshold mood symptomatology, either as mood symptoms or as categorical diagnoses, has been associated with higher risk of conversion in several prior studies. ${ }^{7,8}$ Girls were also more likely to convert, which is consistent with previous literature showing adolescent girls to be at greater risk for mood disturbances overall when compared to adolescent boys. ${ }^{45}$

An individual risk calculator to predict the 5-year risk of developing bipolar disorder in bipolar offspring has been recently proposed. ${ }^{11}$ This risk calculator includes 
variables identified as prodromal symptoms of bipolar disorder in a recent meta-analysis, ${ }^{28}$ and identified anxiety, manic symptoms, depressive symptoms, mood lability, poor general psychosocial functioning, and earlier parental age at onset as collectively predicting bipolar disorder in bipolar offspring. Despite being a much-needed development in the field, the treatment implications of this tool are currently limited. Given the high prevalence of non-mood psychiatric conditions and the need to use antidepressants, stimulants, or second-generation antipsychotics to treat these conditions in bipolar offspring, it is imperative to investigate how medication exposure might influence the risk of developing major depressive or bipolar disorder in bipolar offspring, in order to create more accurate predictive models.

Several limitations must be considered when interpreting the present results. We did not control for all variables previously found to be associated with bipolar offspring converting to bipolar disorder or major depressive disorder, including stressful life events and substance abuse. We did not assess stability of parental illness; future studies should examine the possible associations between parental illness and offspring risk for/course of bipolar illness. Although the study is prospective, subjects and family members were retrospectively interviewed about past medication exposure, which may have introduced recall bias. The smaller sample size of the converter group, rater bias, and differences in follow-up duration between groups were also limitations, along with lack of blood level monitoring of medication to assess adherence. Finally, we did not investigate the effects of polypharmacy, and the additive or moderating effects of medication and psychotherapy cannot not be ruled out. Thus, these results should be regarded as preliminary, particularly the findings on medication exposure. A prospective double-blind randomized controlled trial comparing conversion rates in bipolar offspring treated with antidepressants would a better design to investigate antidepressant exposure as a risk factor for development of major depressive disorder or bipolar disorder in this population.

In conclusion, there is an urgent need to understand risk factors associated with development of psychiatric disorders among bipolar offspring. Since symptoms frequently emerge in early adolescence, it is important to recognize risk factors before and during this crucial developmental period in order to effectively intervene and potentially prevent poor outcomes in these high-risk offspring. Bipolar offspring are often treated with psychopharmacological medication, but little is known about any potential deleterious or protective effects of medication on risk of developing a first mood episode. In this prospective study of bipolar offspring, recruited prior to and followed up naturalistically until developing a first mood episode, we were able to confirm that the presence of mood symptoms and anxiety disorders are strong predictors of conversion to a mood episode. We also found that antidepressant exposure increases the risk of conversion, even after adjusting for baseline depressive symptoms and anxiety. These findings add to the literature on risk factors for conversion, which may help to build better predictive models, and emphasize the need to develop alternative efficacious treatments and preventative strategies for the development of mood disorders among bipolar offspring.

Clinicians should closely monitor bipolar offspring with depressive symptoms or anxiety disorders, as they are at higher risk of developing major depressive disorder or bipolar disorder. This subset is at ultra-high risk for converting to a mood episode. Additionally, clinicians should also carefully monitor bipolar offspring who are treated with antidepressants, given the possible increased likelihood of conversion to a major depressive disorder or bipolar disorder.

\section{Acknowledgements}

This study was partly supported by a National Institutes of Health (NIH; grant \# 5 P50 MH077138 awarded to SMS) and by NIH/NIMH R32 grant \#MH083924. JRS has received research support from the NIH.

The authors would like to thank the participants and families who were involved in this study.

\section{Disclosure}

FGN's spouse is an employee of Eli Lilly \& Co. JRS has received research support from Edgemont, Allergan, Lundbeck, Neuronetics, Otsuka and Shire; has received material support from Assurex/Genesight; and receives royalties from Springer Publishing for the publication of two textbooks, as well as UpToDate and honoraria from CMEology. CMA has received research support from AstraZeneca, Amylin, Eli Lilly, GlaxoSmithKline, Lundbeck, Martek, Merck, Novartis, Otsuka, Pfizer, Takeda, Forest, Actavis, and Shire; has been on the lecture bureau for Merck and Sunovion, for which he has received honoraria. SMS chairs the Data Safety Monitoring Board (DSMBs) for Sunovion and serves on a DSMB for Otsuka; and has received research support from Janssen. MPDB has received research support from Amylin, Eli Lilly, Pfizer, Otsuka, GlaxoSmithKline, Merck, Martek, Novartis, Lundbeck, Pfizer, Sunovion, and Shire; has received consulting/advisory board/honoraria/travel support from Pfizer, Lundbeck, Sunovian, Supernus, and Otsuka. The other authors report no conflicts of interest.

\section{References}

1 DelBello MP, Geller B. Review of studies of child and adolescent offspring of bipolar parents. Bipolar Disord. 2001;3:325-34.

2 Chang K, Steiner H, Dienes K, Adleman N, Ketter T. Bipolar offspring: a window into bipolar disorder evolution. Biol Psychiatry. 2003;53:945-51.

3 Birmaher B, Axelson D, Monk K, Kalas C, Goldstein B, Hickey MB, et al. Lifetime psychiatric disorders in school-aged offspring of parents with bipolar disorder: the Pittsburgh bipolar offspring study. Arch Gen Psychiatry. 2009;66:287-96.

4 Duffy A, Horrocks J, Doucette S, Keown-Stoneman C, McCloskey S, Grof P. The developmental trajectory of bipolar disorder. Br J Psychiatry. 2014;204:122-8.

5 Mesman E, Nolen WA, Reichart CG, Wals M, Hillegers MH. The Dutch bipolar offspring study: 12-year follow-up. Am J Psychiatry. 2013;170:542-9.

6 Duffy A, Alda M, Crawford L, Milin R, Grof P. The early manifestations of bipolar disorder: a longitudinal prospective study of the offspring of bipolar parents. Bipolar Disord. 2007;9:828-38. 
7 Nurnberger JI Jr, Mclnnis M, Reich W, Kastelic E, Wilcox HC, Glowinski A, et al. A high-risk study of bipolar disorder. Childhood clinical phenotypes as precursors of major mood disorders. Arch Gen Psychiatry. 2011;68:1012-20.

8 Hafeman DM, Merranko J, Axelson D, Goldstein BI, Goldstein T, Monk K, et al. Toward the definition of a bipolar prodrome: dimensional predictors of bipolar spectrum disorders in at-risk youths. Am J Psychiatry. 2016;173:695-704.

9 Henin A, Biederman J, Mick E, Sachs GS, Hirshfeld-Becker DR, Siegel RS, et al. Psychopathology in the offspring of parents with bipolar disorder: a controlled study. Biol Psychiatry. 2005;58:554-61.

10 Singh MK, DelBello MP, Kowatch RA, Strakowski SM. Co-occurrence of bipolar and attention-deficit hyperactivity disorders in children. Bipolar Disord. 2006;8:710-20.

11 Hafeman DM, Merranko J, Goldstein TR, Axelson D, Goldstein BI, Monk K, et al. Assessment of a person-level risk calculator to predict new-onset bipolar spectrum disorder in youth at familial risk. JAMA Psychiatry. 2017;74:841-7.

12 Reichart CG, Nolen WA. Earlier onset of bipolar disorder in children by antidepressants or stimulants? An hypothesis. J Affect Disord. 2004;78:81-4.

13 Goldstein BI, Shamseddeen W, Axelson DA, Kalas C, Monk K, Brent DA, et al. Clinical, demographic, and familial correlates of bipolar spectrum disorders among offspring of parents with bipolar disorder. J Am Acad Child Adolesc Psychiatry. 2010;49:388-96.

14 Strawn JR, Adler CM, McNamara RK, Welge JA, Bitter SM, Mills NP et al. Antidepressant tolerability in anxious and depressed youth at high risk for bipolar disorder: a prospective naturalistic treatment study. Bipolar Disord. 2014;16:523-30.

15 Chang KD, Saxena K, Howe M, Simeonova D. Psychotropic medication exposure and age at onset of bipolar disorder in offspring of parents with bipolar disorder. J Child Adolesc Psychopharmacol. 2010;20:25-32.

16 Nery FG, Weber WA, Blom TJ, Welge J, Patino LR, Strawn JR, et al. Longitudinal proton spectroscopy study of the prefrontal cortex in youth at risk for bipolar disorder before and after their first mood episode. Bipolar Disord. 2019;21:330-41.

17 Zhang W, Nery FG, Tallman MJ, Patino LR, Adler CM, Strawn JR, et al. Individual prediction of symptomatic converters in youth offspring of bipolar parents using proton magnetic resonance spectroscopy. Eur Child Adolesc Psychiatry; 2020 Feb 1. doi: 10.1007/ s00787-020-01483-x. Online ahead of print.

18 Nery FG, Norris M, Eliassen JC, Weber WA, Blom TJ, Welge JA, et al. White matter volumes in youth offspring of bipolar parents. J Affect Disord. 2017;209:246-53.

19 First MB, Gibbon M, Spitzer RL, Williams JBW. Structured clinical interview for DSM-IV-TR axis I disorders. New York: State Psychiatic Institute, Biometrics Research Department; 2002.

20 Kaufman J, Birmaher B, Brent D, Rao U, Flynn C, Moreci P, et al. Schedule for affective disorders and schizophrenia for schoolage children-present and lifetime version (K-SADS-PL): initial reliability and validity data. J Am Acad Child Adolesc Psychiatry. 1997;36: 980-8.

21 Geller B, Zimerman B, Williams M, Bolhofner K, Craney JL, DelBello $\mathrm{MP}$, et al. Reliability of the Washington University in St. Louis kiddie schedule for affective disorders and schizophrenia (WASH-UKSADS) mania and rapid cycling sections. J Am Acad Child Adolesc Psychiatry. 2001;40:450-5.

22 Hamilton M. Hamilton psychiatric rating scale for depression. In: Guy W, editor. ECDEU assessment manual for psychopharmacology, revised. Washington: US Department of Health, Education, and Welfare; 1976. p. 179-92.

23 Young RC, Biggs JT, Ziegler VE, Meyer DA. A rating scale for mania: reliability, validity and sensitivity. Br J Psychiatry. 1978;133:429-35.

24 Guy W. Clinical global impressions. In: Guy W editor. ECDEU assessment manual for psychopharmacology, revised. Washington: US Department of Health, Education, and Welfare; 1976. p. 217-22.

25 Keller MB, Lavori PW, Friedman B, Nielsen E, Endicott J, McDonaldScott $P$, et al. The longitudinal interval follow-up evaluation. A comprehensive method for assessing outcome in prospective longitudinal studies. Arch Gen Psychiatry. 1987;44:540-8.

26 Reichart CG, van der Ende J, Wals M, Hillegers MH, Nolen WA, Ormel J, et al. The use of the $\mathrm{GBI}$ as predictor of bipolar disorder in a population of adolescent offspring of parents with a bipolar disorder J Affect Disord. 2005;89:147-55.

27 Duffy A, Horrocks J, Doucette S, Keown-Stoneman C, McCloskey S, Grof P. Childhood anxiety: an early predictor of mood disorders in offspring of bipolar parents. J Affect Disord. 2013;150:363-9.

28 Van Meter AR, Burke C, Youngstrom EA, Faedda GL, Correll CU. The bipolar prodrome: meta-analysis of symptom prevalence prior to initial or recurrent mood episodes. J Am Acad Child Adolesc Psychiatry. 2016;55:543-55

29 Miklowitz DJ, Schneck CD, Walshaw PD, Singh MK, Sullivan AE, Suddath RL, et al. Effects of family-focused therapy vs enhanced usual care for symptomatic youths at high risk for bipolar disorder: a randomized clinical trial. JAMA Psychiatry. 2020;77:455-63.

30 Cotton S, Kraemer KM, Sears RW, Strawn JR, Wasson RS, McCune $\mathrm{N}$, et al. Mindfulness-based cognitive therapy for children and adolescents with anxiety disorders at-risk for bipolar disorder: a psychoeducation waitlist controlled pilot trial. Early Interv Psychiatry. 2020; 14:211-9.

31 Lagopoulos J, Malhi G. Impairments in "top-down" processing in bipolar disorder: a simultaneous fMRI-GSR study. Psychiatry Res. 2011;192:100-8.

32 Iordan AD, Dolcos S, Dolcos F. Neural signatures of the response to emotional distraction: a review of evidence from brain imaging investigations. Front Hum Neurosci. 2013;7:200.

33 Tao R, Calley CS, Hart J, Mayes TL, Nakonezny PA, Lu H, et al. Brain activity in adolescent major depressive disorder before and after fluoxetine treatment. Am J Psychiatry. 2012;169:381-8.

$34 \mathrm{Fu} \mathrm{CH}$, Williams SC, Cleare AJ, Brammer MJ, Walsh ND, Kim J, et al. Attenuation of the neural response to sad faces in major depression by antidepressant treatment: a prospective, event-related functional magnetic resonance imaging study. Arch Gen Psychiatry. 2004;61: 877-89.

35 Norbury R, Taylor MJ, Selvaraj S, Murphy SE, Harmer CJ, Cowen PJ. Short-term antidepressant treatment modulates amygdala response to happy faces. Psychopharmacology (Berl). 2009;206:197-204.

36 Di Simplicio M, Norbury R, Reinecke A, Harmer CJ. Paradoxical effects of short-term antidepressant treatment in fMRI emotional processing models in volunteers with high neuroticism. Psychol Med. 2014;44:241-52.

37 Ladouceur CD, Diwadkar VA, White R, Bass J, Birmaher B, Axelson $\mathrm{DA}$, et al. Fronto-limbic function in unaffected offspring at familial risk for bipolar disorder during an emotional working memory paradigm. Dev Cogn Neurosci. 2013;5:185-96.

38 Chang K, Adleman NE, Dienes K, Simeonova DI, Menon V, Reiss A Anomalous prefrontal-subcortical activation in familial pediatric bipolar disorder: a functional magnetic resonance imaging investigation. Arch Gen Psychiatry. 2004;61:781-92.

39 Drabant EM, Hariri AR, Meyer-Lindenberg A, Munoz KE, Mattay VS, Kolachana BS, et al. Catechol O-methyltransferase val158met genotype and neural mechanisms related to affective arousal and regulation. Arch Gen Psychiatry. 2006;63:1396-406.

40 Gogtay N, Giedd JN, Lusk L, Hayashi KM, Greenstein D, Vaituzis AC, et al. Dynamic mapping of human cortical development during childhood through early adulthood. Proc Natl Acad Sci U S A. 2004;101:8174-9.

41 Lubman DI, Cheetham A, Yucel M. Cannabis and adolescent brain development. Pharmacol Ther. 2015;148:1-16.

42 DelBello MP, Soutullo CA, Hendricks W, Niemeier RT, McElroy SL, Strakowski SM. Prior stimulant treatment in adolescents with bipolar disorder: association with age at onset. Bipolar Disord. 2001;3: 53-7.

$43 \mathrm{Kim}$ JW, Yu H, Ryan ND, Axelson DA, Goldstein BI, Goldstein TR et al. Longitudinal trajectories of ADHD symptomatology in offspring of parents with bipolar disorder and community controls. J Clin Psychiatry. 2015;76:599-606.

44 DelBello MP, Hanseman D, Adler CM, Fleck DE, Strakowski SM Twelve-month outcome of adolescents with bipolar disorder following first hospitalization for a manic or mixed episode. Am J Psychiatry. 2007;164:582-90.

45 Costello EJ, Angold A, Burns BJ, Stangl DK, Tweed DL, Erkanli A, et al. The great smoky mountains study of youth. Goals, design, methods, and the prevalence of DSM-III-R disorders. Arch Gen Psychiatry. 1996;53:1129-36. 